\title{
Perspective
}

PERSPECTIVE Actualité en histoire de l'art

$4 \mid 2006$

La monographie d'artiste

\section{Les aventures de la monographie de cinéma : le Rossellini de Gallagher}

Hervé Joubert-Laurencin

\section{OpenEdition}

1 Journals

Édition électronique

URL : http://journals.openedition.org/perspective/4231

DOI : 10.4000/perspective.4231

ISSN : 2269-7721

Éditeur

Institut national d'histoire de l'art

\section{Édition imprimée}

Date de publication : 31 décembre 2006

Pagination : 646-649

ISSN : 1777-7852

\section{Référence électronique}

Hervé Joubert-Laurencin, «Les aventures de la monographie de cinéma : le Rossellini de Gallagher », Perspective [En ligne], 4 | 2006, mis en ligne le 31 mars 2018, consulté le 01 octobre 2020. URL : http:// journals.openedition.org/perspective/4231 ; DOI : https://doi.org/10.4000/perspective.4231 


\section{Les aventures de la monographie de cinéma : le Rossellini de Gallagher} Hervé Joubert-Laurencin

Lesaventuresde Roberto RossellinideTag Gallagher, somme monographique qui vient de connaître une admirable édition française (1998 ; Léo Sheer, Paris, 2006), ne cache, comme son titre l'indique, aucune des conquêtes féminines de l'Italien, ni aucune de ses vicissitudes biographiques et filmiques (une carrière en dents de scie, de pirate international ; fig. 1). L'entreprise, très ambitieuse, met à nu, au-delà de Rossellini, toutes les qualités nécessaires à une grande biographie, mais aussi les défauts objectifs de cet objet encore rare et incomplet qu'est, pourrait être ou sera la monographie d'un réalisateur de films. Le volume qui dépasse les mille pages serrées (plus de trois millions de signes) constitue donc un nouveau monument d'érudition rossellinienne aussi contestable dans le détail et les partis pris qu'incontournable pour l'information collectée, fruit de vingt ans de travail, d'une centaine d'entretiens, d'une fréquentation cinéphilique apparemment sans failles de l'intégrale des ouvres et d'une connaissance de la littérature critique. Pour qu'un tel travail puisse exister, il faut que beaucoup ait auparavant été dit, écrit, recherché, mis au jour, collecté et programmé, ce qui n'a pas si souvent un caractère d'évidence pour les cinéastes : première réalité spécifique à l'histoire de l'art cinématographique.

Aussi les sources d'information qui sont aujourd'hui à la disposition de l'honnête homme pour confirmer, contester ou compléter la monographie de Gallagher sont-elles nombreuses. Michèle Lagny, dans un article récent très informé publié dans la revue $C i$ néma 011, a fait le point sur les connaissances de plus en plus précises dont nous disposons aujourd'hui quant à la

1. Tag Gallagher, Les aventures de Rossellini,page de couverture.

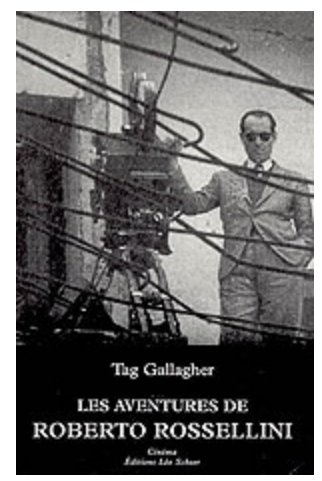

sa vie, de 1963 (l'année même d'une certaine fin d'Hollywood, que le mythe Rossellini a pu effectivement figurer) à 1977. Le réalisateur de Rome ville ouverte et de Voyage en Italie, d'Allemagne année zéro et de La machine à tuer les méchants réalisa des films pendant quarante ans exactement, depuis ses premiers pas en 1937, à l'âge de 31 ans ("Jusque-là il avait toujours vécu capricieusement, au jour le jour ", selon le témoignage de sa première femme cité par Gallagher p. 73), jusqu'à son dernier documentaire, Le Centre Georges-Pompidou, diffusé par l'ORTF le 4 juin 1977, lendemain de sa mort. Une première importante rétrospective des films de Rossellini pour la télévision a été organisée en 2001 à l'Auditorium du Louvre par Philippe-Alain Michaud, accompagnée d'un colloque sur le sujet et de la sortie d'un ouvrage de textes de l'auteur choisis et présentés par le spécialiste italien de Rossellini, Adriano Aprà ${ }^{1}$. Une rétrospective intégrale à la Cinémathèque française début 2006 a complété les possibilités matérielles de voir les œuvres dans les conditions de l'art. Seconde spécificité du cinéma : un effort monographique prend du sens lorsqu'un grand nombre et, dans l'idéal comme c'est ici le cas, plutôt exceptionnel, tous les films d'un cinéaste deviennent visibles en salle. Rétrospective sur plusieurs semaines, mais aussi rachat massif de droits par un même distributeur et/ou retirage, voire restauration de copies anciennes, le plus souvent à l'occasion d'un festival de cinéma : la visibilité des œuvres n'est pas donnée si facilement et l'institution du livre de cinéma n'est pas encore historiquement assez forte pour la provoquer, ni même aussi bien l'accompagner qu'un catalogue de peinture d'un grand musée. Après le ou les livres et la projection systématique en salles, la troisième existence des œuvres, qui est à la fois une origine et un aboutissement de la monographie de cinéma, est la reproduction domestique manipulable de l'œuvre, techniquement assurée il y a peu de temps encore sur cassette vidéo enroulable, et désormais - cela change plus de choses qu'on ne pourrait le croire - sur disque numérisé : le DVD chapitré et sur lequel des repères peuvent être inscrits, lisible à volonté, à partir de n'importe quel moment du film, sur un écran d'ordinateur ou de télévision. Une intégrale en DVD peut désormais changer la connaissance traditionnelle d'un cinéaste $^{2}$. La projection en salle est une chose, une expérience particulière, qui fait sentir et comprendre un auteur d'une certaine façon. Visionner un 


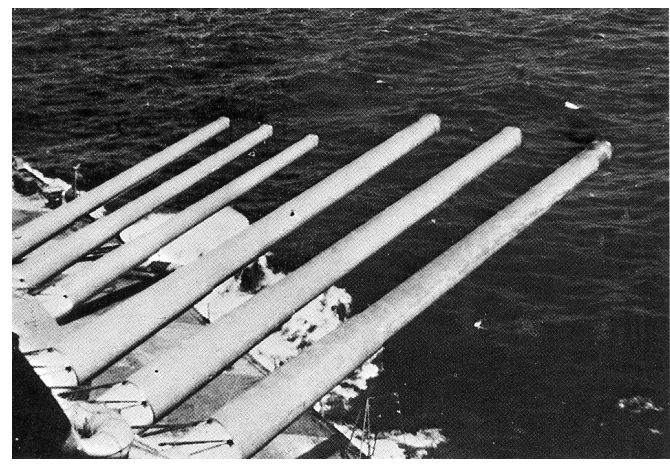

film à loisir, voir et revoir, segmenter, dans une démarche professionnelle ou savante d'analyste de film comme dans une démarche joueuse de spectateur ordinaire (la " nouvelle cinéphilie ") le fait ressentir d'une autre manière ${ }^{3}$. Vivre le cinéma par la mémoire est une troisième approche, tout aussi valide que les précédentes, qui existe, comme la première, depuis l'invention du cinéma, et que le livre de cinéma encourage et relance. Les trois connaissances sont en soi complètes et suffisantes : d'où les problèmes spécifiques que pose la monographie de cinéma, lieu d'écartèlement écrit de ces trois réalités.

Prenons maintenant un exemple paradigmatique. On y voit à l'œuvre la contradiction historique de ces trois expériences de spectateur dans l'écriture monographique.

Tag Gallagher a écrit, à l'aide de sa mémoire spécialisée, représentative d'une certaine cinéphilie traditionnelle (autrement dit forgée dans les années 1950-1960), deux chapitres sur les films de Rossellini de la période fasciste : les chapitres 4, "Enfin au travail ", et 5, "La trilogie de la guerre " (p. 72-132). Est ici décrite, film par film, parfois séquence par séquence, la première des « quatre vies " du cinéaste Rossellini identifiées par Alain Bergala, dans son éditorial du programme de la rétrospective du centenaire à la Cinémathèque française ${ }^{4}$. Après la période fasciste, vient la «néoréaliste ", puis celle des " Bergman-films ", enfin celle de la " télévision éducative ". L'enjeu historiographique classique concernant la première période relance, pour le cinéma, le traditionnel débat des relations entre ouvre et position politique objective de l'artiste. En 2006, la génération cinéphile qui a admiré le troisième Rossellini, celle de Gallagher et de Bergala, s'est fait un devoir de rendre

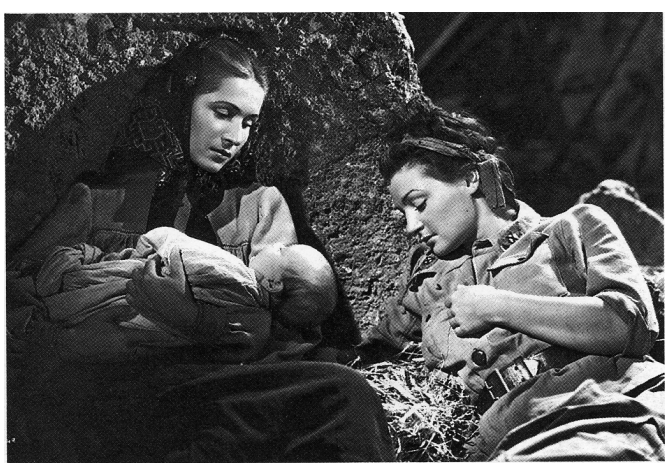

2. Roberto Rossellini, photogramme de La nave bianca.

3. Roberto Rossellini, photogramme de L'uomo della croce. politiquement correct le premier. Ainsi, Bergala écrit-il, au nom de la Cinémathèque française, et en reprenant les arguments de Gallagher : "Cette première période du cinéma de Rossellini consiste en trois films de guerre (la guerre navale dans $\mathrm{La}$ nave bianca [fig. 2], la guerre aérienne dans Un pilota ritorna et la guerre terrestre dans L'uomo della croce [fig. 3]). Le débat a été ravivé à sa mort sur une question morale et politique : Rossellini a-t-il été un 'cinéaste du régime', un propagandiste de la guerre fasciste avant de se convertir par opportunisme à un cinéma de la résistance ? Quiconque voit aujourd'hui sans idées préconçues ces films où le talent du cinéaste est déjà évident, peut juger de lui-même de l'invalidité de cette caricature " ${ }^{5}$. Suivent des arguments, identiques à ceux de Gallagher, sur la peinture réaliste de la guerre qui constituerait un gage antifasciste. L'argumentation défensive culmine, dans Les aventures (p. 106-108), à propos de La nave bianca. Les détails, donnés pas à pas, y contestent des arguments écrits "contre " Rossellini en produisant de brèves ekphrasis censées les détruire, c'est-à-dire du décrit "pour ».

J'ai d'abord lu ces pages, ainsi que celles concernant les autres films, puis j'ai vu, dans le présent d'un spectateur de salle de 2006, les films en question à la Cinémathèque, et alors j'ai relu. Or, voici l'étonnant résultat de mon expérience.

Premier temps: la lecture sans avoir vu les films m'a sincèrement convaincu du bien fondé des arguments de Gallagher, notamment dans ces deux pages et demie sur La nave bianca. L'argumentation pourrait n'être fondée que sur la mémoire cinéphilique de l'auteur. Mais, deuxième temps, toujours à la lecture : bonne surprise, les arguments sont si détaillés que c'est l'analyse de film qui semble y damer le pion à la critique traditionnelle, littéraire et imprécise. La bonne surprise 
consiste donc apparemment en un renouvellement du mode d'écriture de l'histoire du cinéma, sous la pression concertée de l'avancée de l'art de l'analyse de films - enseignée dans toutes les universités depuis maintenant trente ans - et de l'aventure éditoriale monographique. Malheureusement, troisième temps: l'expérience de la vision-audition des films en salle, sans qu'aucune vision supplémentaire sur DVD ne s'avère nécessaire, m'a persuadé aisément et définitivement du contraire : la trilogie est tout simplement la forme du cinéma de guerre mussolinien ; la langue propre du fascisme, son exaltation de la violence et de la mort, trouvent en Rossellini son versant italien doloriste, que les trois films reflètent au mieux ; quelle que soit la polysémie des détails de La nave bianca, pour continuer l'exemple, il faut bien admettre en sortant de la salle que le bateau " blanc " n'est pas neutre, car ce navire-hôpital n'est que l'utopie fasciste d'une jouissance quasi-masochiste des blessures guerrières, dans un confort à la fois fraternel et féminin. La mémoire immédiate du spectacle m'a permis de constater un étrange effet de distorsion : tous les arguments de Gallagher se retournaient, sans que la description des détails pris un par un devienne, en soi, mensongère.

Ainsi, de la nouvelle façon de voir les films (focalisée sur les détails et les petites parties revues plusieurs fois : la cinéphilie DVD), l'argumentation défensive de Gallagher (cependant techniquement issue - on le perçoit à la lecture - d'une vision en salle) ne retient que le grossissement déformant. L'erreur méthodologique de cette utilisation purement verbaliste de l'analyse de film est qu'elle se croit séparée de l'écriture, c'est-à-dire du style du récit monographique lui-même. Elle emporte ainsi l'accumulation de petits faits, interprétés abusivement à leur niveau micrologique, vers une contre-vérité générale flagrante, qu'une seule vision en salle condamne sans appel. On peut ajouter qu'en la matière, une discussion télécommande en main devant une copie DVD, qui suivrait la pente d'un extrémisme scientiste qui n'est pas celui de Gallagher, deviendrait vite oiseuse. En revanche, la copie domestique permet aussi, au-delà de l'accès à des morceaux choisis, de revoir à volonté l'œuvre complète, vision longtemps soumise, pour ces films rares, au hasard d'exceptionnelles rétrospectives.

La distorsion dont j'ai parlé ici n'est pas le simple reflet d'une opinion contre une autre (la mienne contre celle de Gallagher), mais aussi une objective contradiction à la monographie de Gallagher elle-même lorsqu'elle recourt à des éléments issus d'une recherche historique plus traditionnelle. Nombreuses et objectives sont, de fait, les preuves données par Gallagher de l'implication "de régime " de Rossellini : l'adhésion au Parti, les manœuvres pour travailler dans une industrie surveillée, les relations amicales au plus haut niveau; un témoignage sans ambiguïté sur la capacité de Rossellini à " vivre ses mensonges " clôt même le chapitre. Après tout cela, fournir une analyse de trois films qui vise à "innocenter " l'auteur par les détails revient à écrire une seconde histoire dans l'histoire, en comptant sur les films et le génie de l'artiste pour fournir les nouvelles preuves. Au fond, le révisionnisme objectif de Tag Gallagher n'est ici qu'une naïveté théorique.

Même s'il est aussi vrai que le monographe va parfois plus loin dans sa révision lorsqu'il entend se faire historien généraliste. Il tente en effet, par ailleurs, à plusieurs reprises dans son ouvrage, de prouver que le fascisme italien historique était moins fasciste que les démocraties qui lui étaient contemporaines, à travers des proclamations générales qui frisent le ridicule. Benedetto Croce, trop rapidement mais souvent résumé dans le volume, fait office d'idéologue majeur à sauver à tout prix, et y représente l'Italie éternelle. Je rappellerai seulement que Croce, lestement rattaché à Gramsci (p.379), est tenu par celui-ci pour le représentant parfait du " transformisme ", et " la plus puissante machine pour 'conformer' les forces nouvelles aux intérêts vitaux du groupe dominant ". Pourquoi donc ne pas théoriser l'inégalé et nouveau " transformisme " de Rossellini, capable d'incarner presque à lui seul, en trois films, le cinéma de guerre fasciste alors qu'il n'est d'abord qu'un amateur éclairé, puis le cinéma de résistance le plus admirable de l'après-guerre malgré les extraordinaires difficultés matérielles dues à la défaite de son pays ? Voire considérer la dimension de "trahison " constitutive du cinéma moderne qu'il fondera indubitablement au moment même où il épouse une star hollywoodienne ? Le renversement, le divorce sont les qualités et l'ivresse majeures de l'œuvre rossellinienne. C'est même la raison pour laquelle le lecteur de ses aventures est séduit et constamment emporté avec passion sur les pas de cet homme impossible et terriblement humain, de la première à la dernière page. Un tel caractère ne réclame pas une postérité qui le sauve. 
La monographie doit aussi parfois accepter de perdre son auteur, pour ne pas le perdre de vue.

1. Roberto Rossellini, (Adriano Aprà éd.), La télévision comme utopie, Paris, 2001.

2. Citons, dans l'actualité, la stupéfiante intégrale Norman McLaren éditée en 2006 par l'Office national du film du Canada (une exception historique puisque l'Office fut presque l'unique producteur de McLaren, ainsi restaurations et sorties d'inédits, de chutes et de tests filmés complètent-ils une intégrale véritable), et le grand nombre de films de Fassbinder dans un coffret de 18 DVD, rassemblés par la société française de distribution Carlotta Films.

3. Une chercheuse, qui a scruté plan par plan et ligne après ligne (entamant au passage sérieusement le mythe des films sans scénario, entretenu par l'auteur) l'ensemble des six films réalisés avec Ingrid Bergman (1951-1954), considérés comme un "polyptyque " qui fait sens, historiquement audelà des deux personnes en jeu (un rapport entre le nouvel actorat et la modernité), recense même patiemment, par exemple, entre autres documents utiles, les différentes éditions de cassettes vidéo qui témoignent des copies films qui ont pu circuler, avec leurs coupes ou leurs ajouts. Voir la plus philologique des monographies rosselliniennes : Elena Dagrada, Le variente trasparenti. I film con Ingrid Bergman di Roberto Rossellini, Milan, 2005, 478 p., illustrations noir et blanc, et six films découpés systématiquement pour le texte et l'image avec tableaux comparatifs de toutes les versions existantes.

4. Alain Bergala, "Les quatre vies de cinéma de Rossellini ", programme de la Cinémathèque française, janvier-février 2006, p. 28-31.

5. Ibidem.

Hervé Joubert-Laurencin, université Paris 7 - Denis Diderot, hjl@noos.fr

\section{Un voyage dans la lumière : Rainer Werner Fassbinder}

\section{Marianne Dautrey}

En 2005, le Centre George-Pompidou organisait une rétrospective complète de l'œuvre cinématographique de Rainer Werner Fassbinder. À cette occasion, a été traduite la monographie que Thomas Elsaesser avait consacrée au cinéaste, Fassbinder, un cinéaste d'Allemagne (Paris, 2005), tandis qu'en 2005-2006 les éditions Carlotta publiaient une série de quatre coffrets DVD qui, désormais, met à disposition 25 films de Fassbinder, accompagnés de courts-métrages de l'auteur, de documentaires sur ses films et d'extraits d'interviews (fig. 1). La monographie de Thomas Elsaesser marque la tentative d'embrasser l'ensemble des films de Fassbinder à l'intérieur d'un discours spéculatif et cohérent. Les coffrets, eux, présentent sinon l'intégralité de l'œuvre du cinéaste, du moins une sélection de films suffisamment variée pour esquisser une vision d'ensemble de celle-ci. Ne pouvant être autre chose qu'une juxtaposition de films et ne pouvant produire un discours immédiatement cohérent, ils affrontent à l'évidence un problème épineux : celui de faire percevoir les lignes de force qui traversent ces films et la trame qui se tisse de l'un à l'autre au travers d'une simple présentation de ces derniers. Les enjeux de chacune de ces deux présentations de la filmographie de Fassbinder se rejoignent donc : il s'agit de faire apparaître cette dernière comme une ouvre dans son sens plein. Et, dans cette perspective, elles se complètent aussi, se confirmant ou se corrigeant, et permettent un regard nouveau sur le travail du cinéaste.

Le travail du cinéaste Fassbinder fait œuvre d'une manière radicale et volontaire. Cela n'avait pourtant rien eu d'évident jusqu'ici. D'abord parce que ses films traversent des genres parfaitement hétérogènes, tantôt films noirs, tantôt mélodrames, tantôt films sur

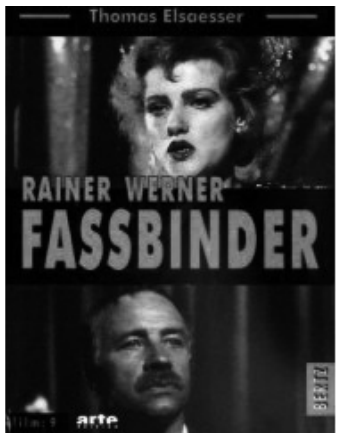

1. Couverture du coffret DVD Fassbinder. 